\title{
Seebeck Coefficients in Nanoscale Junctions: Effects of Electron-vibration Scattering and Local Heating.
}

\author{
Bailey C. Hsu ${ }^{1}$, Yu-Shen Liu ${ }^{1,4}$, Sheng Hsien Lin ${ }^{2,3}$, and Yu-Chang Chen 1 * \\ ${ }^{1}$ Department of Electrophysics, National Chiao Tung University, 1001 Ta Hsueh Road, Hsinchu 30010, Taiwan \\ ${ }^{2}$ Department of Applied Chemistry, National Chiao Tung University, 1001 Ta Hsueh Road, Hsinchu 30010, Taiwan \\ 3 Institute of Atomic and Molecular Sciences, Academia Sinica, Taipei 106, Taiwan \\ 4 College of Physics and Engineering, Changshu Institute of Technology, Suzhou, 215500, China
}

\begin{abstract}
We report first-principles calculations of inelastic Seebeck coefficients in an aluminum monatomic junction. We compare the elastic and inelastic Seebeck coefficients with and without local heating. In the low temperature regime, the signature of normal modes in the profiles of the inelastic Seebeck effects is salient. The inelastic Seebeck effects are enhanced by the normal modes, and further magnified by local heating. In the high temperature regime, the inelastic Seebeck effects are weakly suppressed due to the quasi-ballistic transport.
\end{abstract}

The electron-vibration interaction plays an important role in molecular electronics. Electrons flowing in nanojunctions are characterized by quasi-ballistic electron transport [1]. Only a small fraction of electrons experience the inelastic scattering. Electron-vibration interactions cause discontinuities in the current-voltage (I-V) characteristics known as the inelastic current tunneling spectroscopy (IETS) [2]. The IETS can provide information on the underlying atomic structures of junctions 3 . It also gives important signals to the molecular junction characterization [4].

Electrons that travel with energies larger than the energy of normal modes can excite corresponding vibrations in the nano-structure anchoring the electrodes. This effect causes local heating in the nano-structure [5] 7 . Heating occurs when electrons exchange energy with the excitation and relaxation of the energy levels of the vibration of the nano-structured object that anchors the electrodes. The heating power is typically within $15 \%$ of the electric power $\left(I V_{B}\right)$ supplied by a battery even at ambient temperatures because of the quasi-ballistic transport. The heat generated in the central wire region is dissipated to the bulk electrodes via phonon-phonon interactions. The heat generation eventually equilibrates the heat dissipation, where the wire region reaches an effective local temperature $T_{w}$ higher than the electrode temperature $T_{e}$. Local temperature depends on several factors: the strength of coupling between electrons and the vibrations, the background temperature, and the thermal current which dissipates heat.

In the last decade, remarkable progress has been achieved in measuring the Seebeck coefficients in nanojunctions [8 11]. These experiments have shed light on the design of possible energy-conversion nano-devices, such as nanoscale refrigerators and power generators [12]. These experiments have also inspired rapid development in the theory of thermoelectric nanojunctions 13 22]. In bulk systems, diffused electrons scattered by phonons can significantly affect the Seebeck coefficient. However, the effects of the quasi-ballistic electrons scattered by vibra- tions of the nano-structure on the Seebeck coefficient are relatively unexplored in nanojunctions. To the best of our knowledge only one report for inelastic Seebeck coefficients in molecular junctions based on model calculations is available in the literature [23]. In this Letter, we investigate inelastic Seebeck coefficients from first-principles calculations.

Following the work of Chen, Zwolak, and Di Ventra 5], the many-body Hamiltonian of the system is $H=H_{e l}+$ $H_{v i b}+H_{e l-v i b}$, where $H_{e l}$ is the electronic part of the Hamiltonian under adiabatic approximations and $H_{v i b}$ is the ionic part of the Hamiltonian, which can be casted into a set of independent simple harmonic oscillators via normal coordinates. The normal mode frequencies are $\omega_{j}$, and $H_{e l-v i b}$ is a part of the Hamiltonian for electronvibration interactions which has the form of,

$$
\begin{aligned}
H_{e l-v i b} & =\sum_{\substack{\alpha, \beta, E_{1}, E_{2}, j \\
i, \mu}}\left(\sum_{\frac{\hbar}{2 M_{i} \omega_{j}}} A_{i \mu, j} J_{E_{1}, E_{2}}^{i \mu, \alpha \beta}\right) \\
& \cdot a_{E_{1}}^{\alpha \dagger} a_{E_{2}}^{\beta}\left(b_{j}+b_{j}^{\dagger}\right),
\end{aligned}
$$

where $\alpha, \beta=\{L, R\} ; M_{i}$ is the mass of the $i$-th atom; $A_{i \mu, j}$ is a canonical transformation between normal and Cartesian coordinates satisfying $\sum_{i, \mu} A_{i \mu, j} A_{i \mu, j^{\prime}}=\delta_{j, j^{\prime}}$; $b_{j}$ is the annihilation operator corresponding to the $j$ th normal mode, and $a^{L(R)}$ is the annihilation operator for electrons; the coupling constant $J_{E_{1}, E_{2}}^{i \mu, \beta}$ between electrons and the vibration of the $i$-th atom in $\mu(=x, y, z)$ component can be calculated as,

$J_{E_{1}, E_{2}}^{i \mu, \alpha \beta}=\int d \mathbf{r} \int d \mathbf{K}_{\|}\left[\Psi_{E_{1} \mathbf{K}_{\|}}^{\alpha}(\mathbf{r})\right]^{*}\left[\partial_{\mu} V^{p s}\left(\mathbf{r}, \mathbf{R}_{i}\right) \Psi_{E_{2} \mathbf{K}_{\|}}^{\beta}(\mathbf{r})\right]$,

where $V^{p s}\left(\mathbf{r}, \mathbf{R}_{i}\right)$ is the pseudopotential representing the interaction between electrons and the $i$-th ion; $\Psi_{E \mathbf{K}_{\|}}^{\alpha(=L R)}(\mathbf{r})$ stands for the effective single-particle wave function of the entire system corresponding to incident electrons propagated from the left (right) electrode. These wave functions are calculated iteratively until convergence and self-consistency are achieved in the frame- 


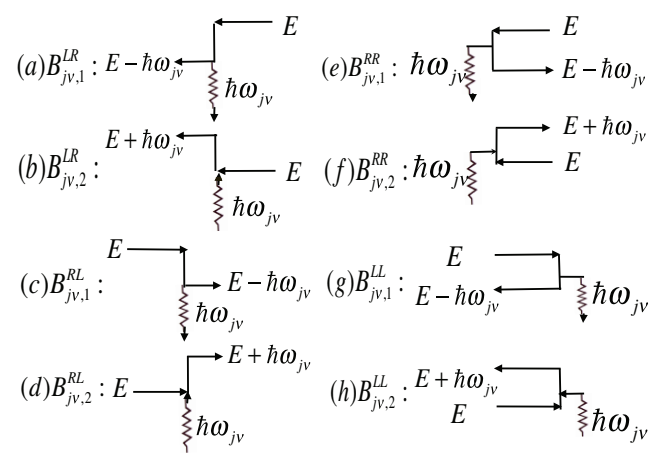

FIG. 1: (color online) Feynman diagrams of the firstorder electron-vibration scattering processes considered in this study.

work of DFT combined with the Lippmann-Schwinger equation 24],

$\Psi_{E \mathbf{K}_{\|}}^{\alpha}(\mathbf{r})=\Psi_{0, E \mathbf{K}_{\|}}^{\alpha}(\mathbf{r})+\int d \mathbf{r}_{1} \int d \mathbf{r}_{2} G\left(\mathbf{r}, \mathbf{r}_{1}\right) V\left(\mathbf{r}_{1}, \mathbf{r}_{2}\right) \Psi_{E \mathbf{K}_{\|}}^{\alpha}$

where $G$ is the Green's function of the biased bimetallic electrodes with $V_{B}=\left(\mu_{R}-\mu_{L}\right) / e$, where $\mu_{R(L)}$ is the chemical potentials deep in the right (left) electrode, respectively; the wave function of the bimetallic junction, $\Psi_{0, E \mathbf{K}_{\|}}^{\alpha}(\mathbf{r})$, is calculated by solving a combination of the Poisson and Schrödinger equations until self-consistency is achieved, where the boundary conditions are given by the electrons deep inside the biased electrodes. The inclusion of a single molecule bridging the bimetallic electrodes is considered as the scattering center, described by the potential $V$.

Our starting point is the inelastic current when considering electron-vibration interactions,

$I\left(\mu_{L}, T_{L} ; \mu_{R}, T_{R} ; T_{w}\right)=\frac{2 e}{h} \int d E\left[\left(f_{E}^{R}-f_{E}^{L}\right)-\left(\tilde{B}^{R}-\tilde{B}^{L}\right)\right] \tau(E)$

where $f_{E}^{L(R)}=1 /\left\{\exp \left[\left(E-\mu_{L(R)}\right) /\left(k_{B} T_{L(R)}\right)\right]+1\right\}$ is the Fermi-Dirac distribution function describing the statistic of electrons deep in the left (right) electrode with temperature $T_{L(R)}$ and chemical potential $\mu_{L(R)}$; the transmission function $\tau(E)=\frac{\pi \hbar^{2}}{m i} \int d \mathbf{R} \int d \mathbf{K}_{\|}\left(\Psi_{E K_{\|}}^{R *} \nabla \Psi_{E K_{\|}}^{R}-\right.$ $\left.\nabla \Psi_{E K_{\|}}^{R *} \Psi_{E K_{\|}}^{R}\right)$ is calculated from the electronic part of the wave functions $\Psi_{E K_{\|}}^{R}$. The terms $\tilde{B}^{L(R)}$ represent the corrections to the elastic current considering the eight first-order scattering processes depicted in Fig. 1.

$\tilde{B}^{\alpha}=\sum_{j}\left[\left\langle\left|B_{j, k}^{\beta, \alpha}\right|^{2}\right\rangle f_{E}^{\alpha}\left(1-f_{E \pm \hbar \omega_{j}}^{\beta}\right)-\left\langle\left|B_{j, k}^{\alpha \alpha}\right|^{2}\right\rangle f_{E}^{\alpha}\left(1-f_{E \pm \hbar \omega_{j}}^{\alpha}\right)\right]$,

where $\alpha, \beta=\{L, R\}$ and $\alpha \neq \beta$. The parameters $B_{j, 1(2)}^{R R}$ and $B_{j, 1(2)}^{L R}$ denoted in Eq. (44) are,

$$
B_{j, 1(2)}^{\alpha R}=i \pi \sum_{i \mu} \sqrt{\frac{\hbar}{2 \omega_{j}}} A_{i \mu, j} J_{E \pm \hbar \omega_{j}, E}^{i \mu, \alpha R} D_{E \pm \hbar \omega_{j}}^{\alpha} \sqrt{\delta+\left\langle n_{j}\right\rangle},
$$

where $\alpha=\{L, R\} ; \delta=0$ (1) represents the process of phonon emission (absorption); the other two parameters in Eq. (44) can be obtained by the relations $B_{j, 1(2)}^{L L}=$ $-B_{j, 1(2)}^{R R}$ and $B_{j, 1(2)}^{R L}=-B_{j, 1(2)}^{L R}$; the average number of local phonons is $\left\langle n_{j}\right\rangle=1 /\left\{\exp \left[\hbar \omega_{j} /\left(k_{B} T_{w}\right)\right]-1\right\}$, where $T_{w}$ is the effective wire temperature.

The rate of energy absorbed (emitted) by the anchored nano-structures due to incident electrons from the $\beta=\{L, R\}$ electrode and scattered to the $\alpha=\{L, R\}$ electrode via a vibrational mode $j$ is denoted by $W_{j}^{\alpha \beta, 2(1)}$. The total thermal power generated in the junction $P$, calculated from the Fermi golden rule, can be written as the sum of all the vibrational modes of eight scattering processes shown in Fig. [1

$$
P=\sum_{j \in v i b} \sum_{\alpha=\{L, R\}} \sum_{\beta=\{L, R\}}\left(W_{j}^{\alpha \beta, 2}-W_{j}^{\alpha \beta, 1}\right) .
$$

The rate of heat dissipated to electrodes via phonon(r) phonon interactions is calculated using the weak link nodel,

$$
J_{p h}=\frac{2 \pi K^{2}}{\hbar} \int_{0}^{\infty} d E E N_{L}(E) N_{R}(E)\left[n_{L}(E)-n_{R}(E)\right],
$$

where $K=1.59 \mathrm{eV} / \mathrm{a}_{0}^{2}$ is the stiffness of the $4-\mathrm{Al}$ atom chain connected to the electrodes obtained from the total energy calculation [25]; $N_{L(R)}(E)$ is the spectral density of local phonon DOS at the left (right) electrode surface from first-principles calculations [26]; and $n_{L(R)} \equiv 1 /\left(e^{E / K_{B} T_{L(R)}}-1\right)$ is the Bose-Einstein distribution function. The effective local temperature $T_{w}$ is obtained when heat generation in the nano-structure and heat dissipation into the bulk electrodes reach balance.

We calculate the inelastic Seebeck coefficient based on the inelastic current described in Eq. (3), which is a func),tion of $T_{L}, T_{R}, T_{w}$, and $V_{B}=\left(\mu_{R}-\mu_{L}\right) / e$. We consider an extra current induced by an infinitesimal temperature difference $(\Delta T)$ across the junction. This current is counterbalanced by an extra current driven by a voltage $(\Delta V)$, which is induced by $\Delta T$ via the Seebeck effect, i.e.,

$$
\begin{aligned}
I\left(\mu_{L}, T_{L} ; \mu_{R}, T_{R}\right) & =\left[I\left(\mu_{L}, T_{L}-\frac{\Delta T}{2} ; \mu_{R}, T_{R}+\frac{\Delta T}{2}\right)\right. \\
& \left.+I\left(\mu_{L}-\frac{e \Delta V}{2}, T_{L} ; \mu_{R}+\frac{e \Delta V}{2}, T_{R}\right)\right] / 2
\end{aligned}
$$

After expanding the above equation to the first order in $\Delta T$ and $\Delta V$, we obtain the inelastic Seebeck coefficient (defined as $S_{e l+v i b}=\Delta V / \Delta T$ ),

$$
S_{e l+v i b}=-\frac{1}{e} \frac{\int d E\left(\frac{\partial \tilde{f}_{E}^{R}}{\partial T_{R}}+\frac{\partial \tilde{f}_{E}^{L}}{\partial T_{L}}\right) \tau(E)}{\int d E\left(\frac{\partial \tilde{f}_{E}^{R}}{\partial E}+\frac{\partial \tilde{f}_{E}^{L}}{\partial E}\right) \tau(E)},
$$

where

$$
\frac{\partial \tilde{f}_{E}^{\alpha}}{\partial E}=\frac{\partial f_{E}^{\alpha}}{\partial E}-\sum_{j \in v i b ; k=1,2}\left(C_{\mu, j, k}^{R \alpha}+C_{\mu, j, k}^{L \alpha}\right)
$$




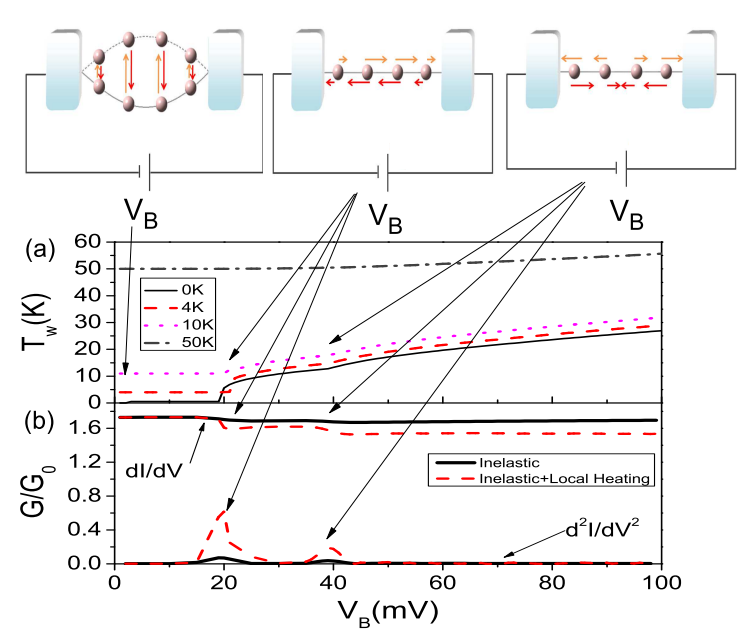

FIG. 2: (color online) (a) Local temperature $T_{w}$ as a function of $V_{B}$ for $T_{e}=0,4,10,50 \mathrm{~K}$; (b) the differential conductance and the absolute value of $d G / d V$ due the electron-vibration interaction without [solid (black) line] and with [dash (red) line] local heating as a function of bias for $T_{e}=12 \mathrm{~K}$. The schematic shows the normal modes that contribute to the jumps in the local temperature and inelastic current profiles.

$$
\frac{\partial \tilde{f}_{E}^{\alpha}}{\partial T_{R}}=\frac{\partial f_{E}^{\alpha}}{\partial T_{R}}-\sum_{j \in v i b ; k=1,2}\left(C_{T, j, k}^{R \alpha}+C_{T, j, k}^{L \alpha}\right),
$$

where $\alpha=\{L, R\}$ and the parameters $C_{\mu, j, 1(2)}^{\alpha R}$ and $C_{T, j, 1(2)}^{\alpha R}$ are,

$C_{\mu, j, 1(2)}^{\alpha R}=\left[f_{E}^{R} \frac{\partial f_{E \pm \hbar \omega_{j \nu}}^{\alpha}}{\partial E}-\left(1-f_{E \pm \hbar \omega_{j}}^{\alpha}\right) \frac{\partial f_{E}^{R}}{\partial E}\right]\left\langle\left|B_{j, 1(2)}^{R R}\right|^{2}\right\rangle ;$

$$
\begin{aligned}
C_{T, j, 1(2)}^{\alpha R} & =\left[\frac{E \pm \hbar \omega_{j}-\mu_{\alpha}}{T_{R}} f_{E}^{R} \frac{\partial f_{E \pm \hbar \omega_{j}}^{\alpha}}{\partial E}\right. \\
& \left.-\frac{E-\mu_{R}}{T_{R}}\left(1-f_{E \pm \hbar \omega_{j}}^{\alpha}\right) \frac{\partial f_{E}^{R}}{\partial E}\right]\left\langle\left|B_{j, 1(2)}^{\alpha R}\right|^{2}\right\rangle,
\end{aligned}
$$

where $\alpha=\{L, R\}$ and $B_{j, 1(2)}^{\alpha \beta}$ are given by Eq. (5). The other two terms in Eq. (9) can be calculated with the following relations $\frac{\partial \tilde{f}_{E}^{L}}{\partial T}=\frac{\partial \tilde{f}_{E}^{R}}{\partial T}(L \rightleftharpoons R)$ and $\frac{\partial \tilde{f}_{E}^{L}}{\partial E}=$ $\frac{\partial \tilde{f}_{E}^{R}}{\partial E}(L \rightleftharpoons R)$, where $L \rightleftharpoons R$ represents the interchange between $R$ and $L$. We see that, in the absence of electronphonon scattering, Eq. (9) recovers the elastic Seebeck coefficient described in Ref. 28.

We now apply our theory to investigating the inelastic Seebeck effects of four $\mathrm{Al}$ atoms bridging the bimetallic $\mathrm{Al}$ electrodes modeled as electron jellium with $r_{s} \approx 2$. The 4-Al junction is structurally and electronically simple such that the first-principle calculations reported here can be performed with a high level of accuracy. It, therefore, serves as an ideal testbed for comparing the predictions of theory and measurements in experiments. We compare the elastic and inelastic Seebeck coefficients assuming that the left and right electrodes share the same
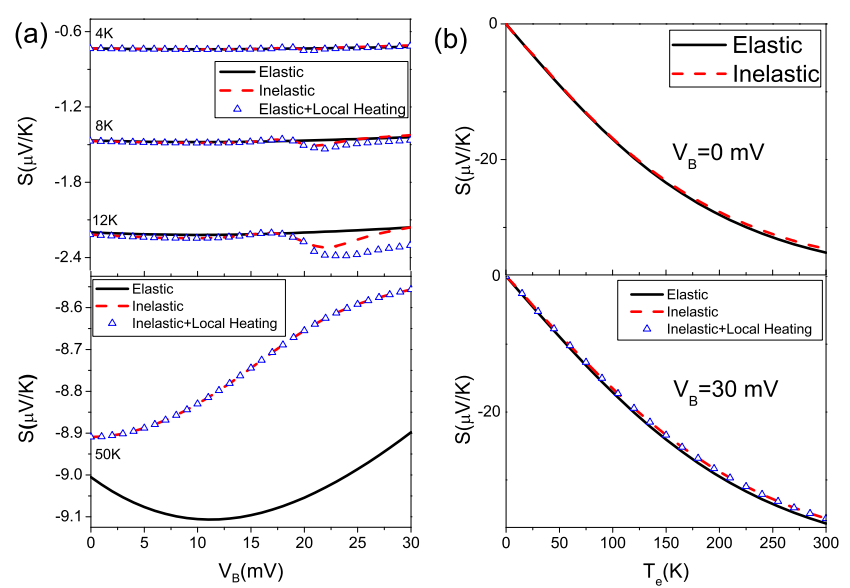

FIG. 3: (color online) Elastic Seebeck coefficient [solid (black) line], inelastic Seebeck coefficient without local heating [dash (red) line], and that with local heating [triangle (blue) line] (a) as a function of bias $V_{B}$ for $T_{e}=4,8,12 \mathrm{~K}$ (in upper panel) and $T_{e}=50 \mathrm{~K}$ (in lower panel); and (b) as a function of $T_{e}$ for $V_{B}=0 \mathrm{~K}$ (in upper panel) and and $V_{B}=30 \mathrm{~K}$ (in lower panel.)

temperature $T_{e}$. In order to qualitatively show to what extent local heating affects the inelastic Seebeck coefficient, we choose to display inelastic Seebeck coefficients with and without local heating.

In the case of "without local heating", we mean that the heat generated in the wire region is perfectly dissipated to electrodes such that $T_{w}=T_{e}$. When including "local heating", the effective local wire temperature $T_{w}$ is higher than the electrode temperature $T_{e}$. Fig. 2(a) shows $T_{w}$ as a function of the applied bias $V_{B}$ for various $T_{e}$. We note that three jumps occur at $V_{B}=2.5,20$, and $40 \mathrm{mV}$, corresponding to the energies of the normal modes. The sharp increase in $T_{w}$ at $V_{B}=20 \mathrm{mV}$ corresponds to the first longitudinal vibrational mode. Two degenerate transverse modes are present in the $x$ - and $y$-directions at $V_{B}=2.5 \mathrm{mV}$, and we show the representative one in Fig. 2. Due to the selection rule, the contributions to local heating from modes with vibrational components perpendicular to the direction of electron transport (z-direction) are unimportant. For $T_{e}=0$, 4 , and $10 \mathrm{~K}, T_{w}$ displays larger jumps at $V_{B}=20 \mathrm{mV}$, where $e V_{B}$ is the energy of the first longitudinal vibrational mode. For $T_{e}=50 \mathrm{~K}$, the signatures of normal modes in $T_{w}$ are wiped out by high temperatures. The increase in local temperature is less significant at higher $T_{e}$. This is due to increasingly efficient heat dissipation caused by the increase of phonon population in the electrodes, as shown in Eq. (77). Fig. 2(b) shows the inelastic profile of the conductance $(G=d I / d V)$ and derivative of conductance $\left(d^{2} I / d V^{2}\right)$ as a function of bias with and without local heating. Local heating enhances the effects of the electron-vibration interactions on the inelastic current because of increased average number of local phonons. 
Figure 3(a) shows Seebeck coefficients as a function of the applied bias $V_{B}$ for various $T_{e}$. For each temperature, we calculate Seebeck coefficients in three cases: elastic Seebeck coefficients $S_{0}$, inelastic Seebeck coefficients without local heating $S_{1}$, and inelastic Seebeck coefficients with local heating $S_{2}$. The difference between the elastic and inelastic Seebeck effects is more salient in the low temperature regime around $V_{B}=20 \mathrm{mV}$ [see the cases of 4,8 , and $12 \mathrm{~K}$ in the upper panel of Fig. 3(a)]. The profile of inelastic Seebeck coefficients vs. $V_{B}$ displays a strong signature corresponding to the longitudinal vibrational mode at $V_{B}=20 \mathrm{mV}$, where the magnitude of the Seebeck coefficients are increased. This feature is related to the suppression of the inelastic current around $V_{B}=20 \mathrm{mV}$ [Fig. 2(b)], where the transmission function effectively decreases. This leads to larger magnitudes of Seebeck coefficients because $S \propto$ $-\tau^{\prime}(\mu) / \tau(\mu)$ [28].

The inclusion of local heating enhances the effect of electron-vibration on Seebeck coefficients further. In the low temperature regime, the upper panel of Fig. 3)(a) shows that $S_{1}$ (without local heating) significantly differs from $S_{2}$ (with local heating). This is because of the large difference between $T_{w}$ and $T_{e}$, as shown in Fig. 2(a). For $V_{B}<30 \mathrm{mV}, T_{w}$ and $T_{e}$ become almost identical when the $T_{e}$ is large. Consequently, the difference between $S_{1}$ and $S_{2}$ becomes small [see cases of $T_{e}=50 \mathrm{~K}$ in the lower panel of Fig. 3(a).] In all cases, the transverse modes are negligible to the inelastic Seebeck coefficients. Fig. 3(b) shows Seebeck coefficients as a function of $T_{e}$ for $V_{B}=0$ and $30 \mathrm{mV}$ in three cases: $S_{0}, S_{1}$, and $S_{2}$. In the high temperature regime $\left(T_{e}>50 \mathrm{~K}\right)$, the magnitudes of inelastic Seebeck coefficients $\left(S_{1}\right.$ and $\left.S_{2}\right)$ are slightly decreased compared with the elastic Seebeck coefficients $\left(S_{0}\right)$ due to small probability of electron-vibration scattering.

In summary, we investigated the elastic and inelastic Seebeck coefficients with and without local heating in the 4-Al atomic junction using first-principles calculations. In the low temperature regime, the signature of normal modes in the profiles of inelastic Seebeck effects is salient. The inelastic Seebeck effects are enhanced by electron-vibration interactions due to the drastic suppression of the inelastic current at the bias corresponding to the normal mode with longitudinal vibrational character. Local heating enhances the inelastic Seebeck effects further due to increased average number of local phonons. In the high temperature regime, the inelastic Seebeck effects are slightly suppressed by electron-vibration interactions due to quasi-ballistic electron transport in nanojunctions. The signature of normal modes in inelastic Seebeck coefficients and local temperatures is wiped out by the tail of the Fermi-Dirac distribution.

The authors thank MOE ATU, NCHC, National Cen- ter for Theoretical Sciences(South) in Taiwan, and NSC (Taiwan) for support under Grants NSC 97-2112-M-009011-MY3. Y. S. thank the support of NSFC under Grant No. 10947130.

* Electronic address: yuchangchen@mail.nctu.edu.tw

[1] M. Galperin, M. A. Ratner, and A. Nitzan, J. Phys.: Condens. Matter. 19, 103201 (2007).

[2] L. H. Yu, C. D. Zangmeister, and J. G. Kushmerick, Phys. Rev. Lett. 98, 206803(2007).

[3] Y. C. Chen, Phys. Rev. B 78, 233310 (2008).

[4] M. Kiguchi et al., Phys. Rev. Lett. 101, 046801 (2008).

[5] Y. C. Chen, M. Zwolak and M. Di Ventra, Nano Lett. 3, 1691 (2003); Nano Lett. 4, 1709 (2004); Nano Lett. 5, 813 (2005).

[6] T. Frederiksen, M. Brandbyge, N. Lorente, and A.P. Jauho, Phys. Rev. Lett. 93, 256601 (2004).

[7] Z. Huang, B. Xu, Y. C. Chen, M. Di Ventra, and N. J. Tao, Nano Lett. 6, 1240 (2006).

[8] B. Ludoph and J. M. van Ruitenbeek, Phys. Rev. B 59, 12290 (1999).

[9] P. Reddy, S. Y. Jang, R. A. Segalman, and A. Majumdar, Science 315, 1568 (2007).

[10] K. Baheti et al., Nano Lett. 8, 715 (2008).

[11] J. A. Malen et al., Nano Lett. 9, 1164 (2009).

[12] Y. S. Liu, H. T. Yao, and Y. C. Chen, condmat/arXiv:1001.0822; cond-mat/arXiv:0908.0992.

[13] M. Paulsson and S. Datta, Phys. Rev. B 67, 241403(R) (2003).

[14] X. Zheng, W. Zheng, Y. Wei, Z. Zeng, and J. Wang, J. Chem. Phys. 121, 8537 (2004).

[15] F. Pauly, J. K. Viljas, and J. C. Cuevas, Phys. Rev. B 78, 035315 (2008).

[16] Y. Dubi and M. Di Ventra, Nano Lett. 9, 97 (2009).

[17] S. H. Ke, W. Yang, S. Curtarolo, and H. U. Baranger, Nano Lett. 9, 1011 (2009).

[18] C. M. Finch, V. M. García-Suárez, and C. J. Lambert, Phys. Rev. B 79, 033405 (2009).

[19] J. P. Bergfield and C. A. Stafford, Nano Lett. 9, 3072 (2009).

[20] Y. S. Liu, Y. R. Chen, and Y. C. Chen, ACS Nano 3, 3497 (2009).

[21] M. Galperin, K. Saito, A. V. Balatsky, and A. Nitzan, Phys. Rev. B 80, 115427 (2009).

[22] T. Markussen, A. P. Jauho, and M. Brandbyge, Phys. Rev. B 79, 035415 (2009).

[23] M. Galperin, A. Nitzan, and M. A. Ratner, Molecular Phys. 106, 397 (2008).

[24] N. D. Lang, Phys. Rev. B 52, 5335 (1995).

[25] Z. Yang, M. Chshiev, M. Zwolak, Y. C. Chen, and M. Di Ventra, Phys. Rev. B 71, 041402(R) (2005).

[26] E.V. Chulkov, I.Yu. Sklyadneva, Surf. Sci. 331, 1414 (1995).

[27] K. R. Patton and M. R. Geller, Phys. Rev. B 64, 155320 (2001).

[28] Y. S. Liu and Y. C. Chen, Phys. Rev. B 79, 193101 (2009). 